\title{
Surgical endodontic treatment under magnification has high success rates
}

\author{
Abstracted from \\ Tsesis I, Faivishevsky V, Kfir A, Rosen E. \\ Outcome of surgical endodontic treatment performed by a modern technique: a meta-analysis of the literature. \\ J Endod 2009; 35: 1505-1511 \\ Address for correspondence: Dr Igor Tsesis, Tel Aviv University Dental School, \\ Maurice and Gabriela Goldschleger School of Dental Medicine, Department of Endodontology, \\ Tel Aviv, Israel. E-mail: dr.tsesis@gmail.com
}

\section{Questions: Is modern surgical endodontic treatment effective and what factors influence the outcome?}

Data sources Medline, Embase and reference lists of identified articles were used to find relevant publications; searches were limited to English language.

Study selection Randomised control trials (RCT) or prospective case series of surgical endodontic treatments performed by a modern technique using magnification, root-end resection with minimal or no bevel, retrograde cavity preparation with ultrasonic tips, and retrograde root canal filling where the lesion was located at the periapical area, with a minimum of 1-year followup and outcomes assessed according to the Rud ${ }^{1}$ or Molven ${ }^{2}$ criteria, were included. Studies that did not use a modern surgical approach, involved teeth with apico-marginal defects, periodontal disease, root fractures and root perforations or undergoing repeat surgery were excluded, as were those with less than 1 -year followup and with outcomes not evaluated using the Rud ${ }^{1}$ or Molven ${ }^{2}$ criteria.

Data extraction and synthesis Studies were reviewed and data extracted independently by three observers, with disagreements resolved by discussion. Studies included were combined to estimate the pooled successes and $95 \%$ confidence intervals $(\mathrm{Cl})$, where success was considered to be complete healing or incomplete healing, by using generalised linear models, assuming a binomial (proportion) distribution.

Results Eleven studies (six RCT and five prospective case series) met the criteria. The pooled proportion of treatments deemed to be a success (complete healing and incomplete healing) was found to be 91.6\% (95\% Cl, 85.9-95.1\%). The pooled proportion deemed to be failures was found to be $4.7 \%(95 \% \mathrm{Cl}, 2.24-9.50 \%)$. The remainder were categorised as of uncertain healing $(3.7 \%)$

Conclusions Surgical endodontic treatment carried out using a modern technique carries predictable outcomes. Additional large-scale prospective clinical studies are needed to evaluate further predictors of success and failure.

\section{Commentary}

The advancement in surgical endodontics (eg, the use of the dental operative microscope, no or minimal bevel of the root-end resection, and ultrasonic retrograde canal preparation to a depth of $3-4 \mathrm{~mm}$ ) has resulted in more precise and predictable surgical procedures, which could not normally be achieved using the traditional surgical endodontic treatment (by means of root-end resection with a 45-degree bevel, retrograde preparation of the canal with bur, and root-end filling under no magnification and inadequate illumination). Therefore, assuming a higher outcome for the modern versus the traditional procedure ${ }^{3}$ this study pooled six RCT and five prospective case-series to evaluate the outcome of the modern surgical endodontic treatment as well as the factors influencing the outcome.

In order to critically appraise this paper, the PRISMA check-

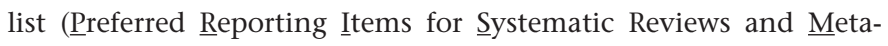
analyses $)^{4}$ was utilised. The search strategy appears to be exhaustive, although it was limited to English publications. The eligibility criteria and data collection process were detailed. Three reviewers worked independently in identifying and appraising the included studies and extracting the data. Such methodology would reduce bias in a review. With regards to the eligibility criteria of the level of evidence and study design, it should be noted that because of the nature of the question (outcome of only modern surgical endodontics), no control group is necessary. Therefore, even though the authors included six RCT, in fact for the purpose of the data synthesis, such RCT had to be handled as prospective case series, ie, only the results of the RCT arm(s) that utilised the modern endodontic surgery procedure and met the other inclusion criteria were considered for the data synthesis.

No report was mentioned of attempting to contact authors of papers that partially met the inclusion criteria for further results (eg, Saunders $2008^{5}$ for the outcome of cases with at least one year followup). Moreover, in some cases, the reasons for excluding a paper seem to be erroneous, since the paper by Gagliani et al. $(2005)^{6}$ was rejected for not using Rud ${ }^{1}$ criteria even though it did so.

There is no indication of risk of bias assessment of individual studies at the study or outcome level (eg, masking and/ or calibration of the examiners) and, thus, no sensitivity analysis was performed for the data synthesis. Moreover, no measure of consistency (eg, $I_{2}$ for testing heterogeneity) ${ }^{7}$ was reported.

In this meta-analysis, the radiographic healing categories of incomplete healing (scar) and complete healing were combined into one category of "success" with a pooled estimate of 91.6\% (95\% 
CI, 85.9-95.1\%). It should be noted that scar tissue (at 1 year) only develops in $<10 \%$ of cases. ${ }^{8-10}$ In contrast, some of the included studies reported an "incomplete healing" as high as 27-50\%, raising the possibility of misclassification of "uncertain healing" or "failure" cases as incomplete healing. Such misclassification would result in an overestimation of the outcome in individual studies, as well as the pooled estimate. Moreover, it should be noted that, in general, $5-25 \%$ of cases classified as healed after 1 year are expected to have a recurrence of peri-apical radiolucency or clinical symptoms at a longer followup. ${ }^{11-13}$ Therefore, the 1-year outcome may not be indicative of a long-term one, again resulting in an overestimate vs the true outcome. Since the termination date of the search strategy, one prospective study ${ }^{14}$ has been published that evaluates the 4-10year outcome of apical surgery. Although this recent publication would not meet the specified inclusion criteria of this meta-analysis (based on the outcome evaluation criteria), it found that only $74 \%$ of 134 examined teeth were "healed" (periapical index score of $\leq 2$ or scar, plus no clinical signs or symptoms).

This meta-analysis found that gender, age, type of root filling material, and magnification technique (operative microscope, endoscope, loupes) did not have any significant impact on the pooled estimate of outcome. Although age was not found to be an outcome predictor in several other studies, a recent prospective study ${ }^{14}$ identified age as a significant predictor of the outcome ( $84 \%$ vs $68 \%$ healing rates in patients older than 45 years or $<45$ years, respectively). There is a need for further investigation of this variable.

In conclusion, this meta-analysis summarised evidence for the short-term (at least 1 year) outcome of modern surgical endodontics. Considering the risk of misinterpreted followup data, the pooled estimate outcome tends to be an overestimation of a true outcome and, thus, should be interpreted with caution.

Amir Azarpazhooh

Community Dental Health Services Research Unit, Faculty of Dentistry, University of Toronto. Toronto, Ontario, Canada
Dr Azarpazhooh thanks Professor Shimon Friedman, Faculty of Dentistry, University of Toronto, for his thoughtful inputs on this commentary.

1. Rud J, Andreasen JO, Jensen JE. Radiographic criteria for the assessment of healing after endodontic surgery. Int / Oral Surg 1972; 1: 195-214.

2. Molven O, Halse A, Grung B. Observer strategy and the radiographic classification of healing after endodontic surgery. Int J Oral Maxillofac Surg 1987; 16: 432-439.

3. Tsesis I, Rosen E, Schwartz-Arad D, Fuss Z. Retrospective evaluation of surgical endodontic treatment: traditional versus modern technique. J Endod 2006; 32: $412-416$.

4. Moher D, Liberati A, Tetzlaff J, Altman DG. Preferred Reporting Items for Systematic Reviews and Meta-analyses: the PRISMA statement. Br Med J 2009; 339: b2535.

5. Saunders WP. A prospective clinical study of periradicular surgery using mineral trioxide aggregate as a root-end filling. J Endod 2008; 34: 660-665.

6. Gagliani MM, Gorni FG, Strohmenger L. Periapical resurgery versus periapical surgery: a 5-year longitudinal comparison. Int Endod J 2005; 38: 320-327.

7. Higgins JP, Thompson SG. Quantifying heterogeneity in a meta-analysis. Stat Med 2002; 21: 1539-1558.

8. Grung B, Molven O, Halse A. Periapical surgery in a Norwegian county hospital: follow-up findings of 477 teeth. J Endod 1990; 16: 411-417.

9. Jensen SS, Nattestad A, Egdø P, Sewerin I, Munksgaard EC, Schou S. A prospective, randomised, comparative clinical study of resin composite and glass ionomer cement for retrograde root filling. Clin Oral Investig 2002; 6: 236-243.

10. Altonen M, Mattila K. Follow-up study of apicoectomized molars. Int J Oral Surg 1976; 5: $33-40$.

11. Yazdi PM, Schou S, Jensen SS, Stoltze K, Kenrad B, Sewerin I. Dentine-bonded resin composite (Retroplast) for root-end filling: a prospective clinical and radiographic study with a mean follow-up period of 8 years. Int Endod J 2007; 40: 493-503.

12. Wesson CM, Gale TM. Molar apicectomy with amalgam root-end filling: results of a prospective study in two district general hospitals. Br Dent / 2003; 195: 707-714.

13. Kvist T, Reit C. Results of endodontic retreatment: a randomized clinical study comparing surgical and nonsurgical procedures. J Endod 1999; 25: 814-817.

14. Barone C, Dao TT, Basrani BB, Wang N, Friedman S. Treatment outcome in endodontics: the Toronto study—phases 3, 4 and 5: apical surgery. J Endod 2010; 36: $28-35$.

\section{Practice points}

- Surgical endodontic treatment under magnification and illumination with no or minimal bevel of root-end resection, ultrasonic retrograde canal preparation to a depth of 3-4 $\mathrm{mm}$, and retrograde root canal filling is a highly successful treatment.

Evidence-Based Dentistry (2010) 11, 71-72. doi:10.1038/sj.ebd.6400733 